\title{
Calculation and Simulation of Chemical-Diffusion Coefficients: The Inadequacy of the Mean-Field Theory
}

\author{
Michael Atzmon \\ Department of Nuclear Engineering, The University of Michigan, Ann Arbor, Michigan 48109
}

(Received 22 March 1990)

\begin{abstract}
Initial chemical-interdiffusion coefficients in a simple cubic, random, binary alloy, Ising model have been determined analytically and by Monte Carlo simulations, using chemical rate equations for atomic exchanges. The results, obtained as a function of the composition gradient, enthalpy of mixing, and temperature, show that the chemical enhancement of the diffusion coefficients saturates and is considerably lower than predicted by Darken's equation. The discrepancy is due to the inadequacy of the mean-field theory and linear-response approximation at high enthalpies of mixing.
\end{abstract}

PACS numbers: $66.30 . \mathrm{Ny}, 64.60 . \mathrm{Cn}, 82.20 . \mathrm{Wt}$

Interdiffusion in concentrated solid alloys has been of interest for many years. In addition to the fundamental interest, there are also practical motivations for its study. Some alloy synthesis methods rely on solid-state diffusion, and the stability of devices with steep composition gradients depends on its absence. One important issue is the effect of chemical interactions between the alloyed species on the magnitude of the diffusion coefficient. Depending on the sign of the enthalpy of mixing per atom, $\Delta H_{\text {mix }}$, the diffusion coefficient will be enhanced, reduced, or even negative, leading to spinodal decomposition.' While previous treatments ${ }^{2}$ are based on the mean-field ${ }^{3}$ (MF) theory and linear-response ${ }^{4}$ (LR) approximation, this Letter presents an atomistic analysis and points out the limited validity of the previously used approach for $\eta=\Delta H_{\text {mix }} / k_{B} T \geq 1$, where $T$ is the temperature and $k_{B}$ is Boltzmann's constant. A significant related result is observed experimentally in multilayers.

Darken $^{2}$ derived an expression for the chemically modified diffusion coefficient, assuming LR, i.e., a proportionality between diffusional flux of species $\alpha, \mathbf{J}_{\alpha}$, and the gradient of its chemical potential $\mu_{\alpha}$ :

$$
\mathbf{J}_{\alpha}=-C_{\alpha} M_{\alpha} \nabla \mu_{\alpha}
$$

where $M_{\alpha}$ is the mobility of the atomic species $\alpha$, and $C_{\alpha}$ its concentration. This and all other equations in this Letter are given in the lattice coordinate system. Expressing $\nabla \mu$ in terms of $c_{\alpha}$, the fraction of sites occupied by $\alpha$ atoms, Darken obtained an expression for the chemical-diffusion coefficient $D_{\alpha}$ :

$$
D_{\alpha}=k_{B} T M_{\alpha}\left(1+\frac{\partial \ln \gamma_{\alpha}}{\partial \ln c_{\alpha}}\right)=D_{\alpha}^{*}\left(1+\frac{\partial \ln \gamma_{\alpha}}{\partial \ln c_{\alpha}}\right),
$$

where $\gamma_{\alpha}$ is the activity coefficient of $\alpha . D_{\alpha}^{*}$ is the tracer-diffusion coefficient of $\alpha-$ it is measured by monitoring the evolution kinetics of an initially nonuniform isotopic distribution of $\alpha$ for a uniform chemical composition. The term in parentheses is called the thermo- dynamic factor. For a regular solution, ${ }^{5}$ Eq. (2) can be expressed as ${ }^{6}$

$$
D_{\alpha}=D_{\alpha}^{*}\left(1-2 \Delta H_{\mathrm{mix}} / k_{B} T\right) \text {. }
$$

The derivation of Eq. (2) from Eq. (1) is exact, but Eq. (1) is approximate because (1) it uses the chemical potential as a mean field ${ }^{3}$ and (2) it is a first-order expansion of $\mathbf{J}$ in $\nabla \mu_{\alpha}$, i.e., a LR approximation. ${ }^{4}$ The latter was assumed to apply when the composition variations between neighboring lattice sites are small, i.e.,

$$
\left|\nabla c_{\alpha}\right| \ll \lambda^{-1}
$$

where $\lambda$ is the interatomic separation. For a finite $\Delta H_{\text {mix }}$ and fixed temperature, Eq. (4) implies that the chemical-potential variations between neighboring lattice sites are small, i.e.,

$$
\lambda\left|\nabla \mu_{\alpha}\right| \ll k_{B} T .
$$

Both the MF theory and the LR approximation are commonly used to describe fluxes in nonuniform systems.

$\mathrm{Tu}^{7}$ has applied chemical rate equations to derive chemical-diffusion coefficients in alloys. His analysis employs the chemical potential and therefore the MF theory, but not the LR approximation. If one normalizes Tu's exponential jump probabilities, his result has the form

$$
J_{\alpha}=-\frac{v}{\lambda^{2}} \tanh \left(\frac{\frac{1}{4} \lambda \nabla \mu_{\alpha}(\mathbf{r})}{k_{B} T}\right) .
$$

$v$ is the rate of successful atomic jumps for unbiased random walk, equal to $v_{0} \exp \left(\Delta E / k_{B} T\right)$, where $v_{0}$ is the rate of atomic attempts to jump over a saddle point of height $\Delta E$. For a given enthalpy of mixing, Eq. (5) is satisfied for a sufficiently small $\nabla_{c}$ [Eq. (4)], and Eq. (6) is then reduced to Darken's assumption, Eq. (1). On the other hand, for a given $\nabla c$, a sufficiently large enthalpy of mixing would lead to a nonlinear dependence of the flux on $\nabla c$. We stress that, within the atomic-jump model, the only approximation used in the derivation of Eq. (6) is 
the MF theory.

More sophisticated analytical methods have been applied to diffusion in alloys (see review in Ref. 8). These were aimed primarily at calculating correlation factors for the vacancy mechanism and did not address the atomistic origin of Darken's enhancement. Murch ${ }^{9}$ has confirmed Darken's equation for a lattice gas by Monte Carlo simulations. However, he did not cover strong interactions ( $\eta \geq 1$ for an alloy), for which a single-phase lattice gas is not in equilibrium.

Beyond the MF theory, if individual atomic jumps are considered, they are expected to lead to a discrete spectrum of enthalpy changes, $\Delta \varepsilon_{i}$, which will depend on the local atomic configurations. Because of local composition fluctuations, the values of $\Delta \varepsilon_{i}$ will not vanish for a vanishing composition gradient. The present Letter demonstrates that this fact has a profound effect on the diffusion behavior. We have modeled alloy diffusion with atomic exchanges in a simple cubic (3D), chemically random, binary alloy, Ising model with conserved dynamics. ${ }^{10}$ We report the results of both exact calculations and Monte Carlo simulations of the initial diffusional flux as a function of $\eta$ and the concentration gradient. The chemical enhancement is shown to saturate at a value lower than 3 , in contrast with an enhancement as high as 100 predicted by Darken's equation, leading to the conclusion that the MF theory fails when $\eta \geq 1$.

We have studied a chemically random alloy on a simple cubic lattice with equal average fractions of $\alpha$ and $\beta$ atoms, a constant composition gradient in the (100) direction, and fixed compositions of the boundaries perpendicular to the gradient. Nearest-neighbor (NN) interactions were assumed, with the bond energies between $x$ and $y, \varepsilon_{x y}$, set as $\varepsilon_{\alpha \alpha}=\varepsilon_{\beta \beta}=-\varepsilon$ and $\varepsilon_{\alpha \beta}=\varepsilon_{\beta \alpha}=\varepsilon$, yielding $\Delta H_{\mathrm{mix}}=3 \varepsilon .^{5}$ Exchanges between randomly selected atoms and their NN's were performed with a probability equal to $\exp \left(-\Delta \varepsilon / k_{B} T\right) /\left[1+\exp \left(-\Delta \varepsilon / k_{B} T\right)\right]$, where $\Delta \varepsilon$ is the energy change upon exchange. The resulting behavior is therefore a function of $\eta$. Atomic exchanges between two neighboring planes were considered, and two additional planes provided the correct NN environment. The steady-state flux, for fixed compositions at the end planes, was determined as a function of both the composition gradient and $\eta$. Only the initial stages of diffusion were analyzed, so that the slowdown of the diffusion kinetics as the alloy orders ${ }^{8.11}$ or phase separates does not enter into the results. In this model, $\alpha$ and $\beta$ atoms have the same atomic volumes, flux magnitudes, and diffusion coefficients, the interdiffusion coefficient ${ }^{12}$ is equal to the atomic-diffusion coefficients, and the Kirkendall effect ${ }^{13}$ is absent.

Both analytical treatment and Monte Carlo simulations were used to analyze the model. Simulations were performed on a lattice with 720000 sites. The atomic flux for a given composition gradient was determined by averaging over 500 runs, each consisting of approximately 0.1 Monte Carlo step per lattice site (see Ref. 14 for details). The error was estimated from the spread of the results. Chemical rate equations ${ }^{15-17}$ were applied to obtain an analytical expression for the total flux of $\alpha$ atoms between planes 2 and 3 :

$$
J=\frac{v}{\lambda^{2}} \sum_{l} \frac{\exp \left(-\Delta \varepsilon_{i} / k_{B} T\right)}{1+\exp \left(-\Delta \varepsilon_{i} / k_{B} T\right)}\left[c_{2}\left(1-c_{3}\right) p_{23}\left(\Delta \varepsilon_{i}\right)-\left(1-c_{2}\right) c_{3} p_{32}\left(\Delta \varepsilon_{l}\right)\right] .
$$

$c_{l}$ is the average fraction of $\alpha$ atoms in plane $i$, and $\lambda$ is the lattice parameter. $v$ is the rate at which a given atom participates in an exchange, and is thermally activated in a way similar to that in Eq. (6). The summation is over the values attainable by $\Delta \varepsilon_{i},\{(-20+4 n) \varepsilon\}$, where $0 \leq n \leq 10$ is an integer, and $p_{23}\left(\Delta \varepsilon_{i}\right)\left[p_{32}\left(\Delta \varepsilon_{i}\right)\right]$ is the probability that, given an $\alpha[\beta]$ atom in plane 2 and a NN of type $\beta[\alpha]$ in plane 3 , the surrounding site occupations are such that the energy change upon atomic exchange is $\Delta \varepsilon_{i}$. The following relations hold: $p_{23}\left(\Delta \varepsilon_{i}\right)$ $=p_{32}\left(-\Delta \varepsilon_{i}\right)$ for all $i$, and $\sum_{i} p_{23}\left(\Delta \varepsilon_{i}\right)=\sum_{l} p_{32}\left(\Delta \varepsilon_{i}\right)$ $=1$. The calculation of $p_{23}$ and $p_{32}$ was based on the average compositions of planes 1-4, taking into account the probabilities of all possible NN configurations for a random alloy. The functional behavior of $\mathrm{Eq}$. (7) is better illustrated when expressed in the form

$$
J=\frac{v}{\lambda^{2}} \sum_{i}\left\{\left(\frac{b_{i}-a_{i}}{2}\right)-\left(\frac{a_{l}+b_{i}}{2}\right) \tanh \left(\frac{\Delta \varepsilon_{i}}{2 k_{B} T}\right)\right\},
$$

where $\quad a_{i}=c_{2}\left(1-c_{3}\right) p_{23}\left(\Delta \varepsilon_{i}\right) \quad$ and $\quad b_{1}=\left(1-c_{2}\right) c_{3}$ $\times p_{32}\left(\Delta \varepsilon_{i}\right)$.
In Fig. 1, the calculated flux $J$ is plotted as a function of the concentration gradient $\left|\nabla C_{a}\right|$ for several values of $\eta$. The curves for all $\eta \geq 5$, and those for all $\eta \leq-5$, are nearly identical to each other. For all $\eta$ 's and for gradients less than 10 at. \% per lattice plane, the flux obtained is proportional to the gradient within an error less than $2 \%$. Fick's first law ${ }^{18}$ is therefore valid and one can define a diffusion coefficient as $D=J /\left|\nabla C_{\alpha}\right|$. Its value is plotted in Fig. 2 as a function of $\eta$ along with the corresponding simulation results and Darken's equation for a regular solution [Eq. (3)]. As verified separately, the tracer-diffusion coefficient for the present model is independent of $\eta$. The good agreement between Eq. (7) and the simulation is not surprising because the former is exact for the initial stages of diffusion. One observes that the chemical enhancement saturates for large $\eta$, and does not exceed 3. For real alloys and temperature ranges in which atomic mobility can be observed, $|\eta|$ can be as high as 50 , so that the thermodynamic factor in Darken's equation can exceed \pm 100 . Figure 2 therefore indicates a significant discrepancy between Darken's and 


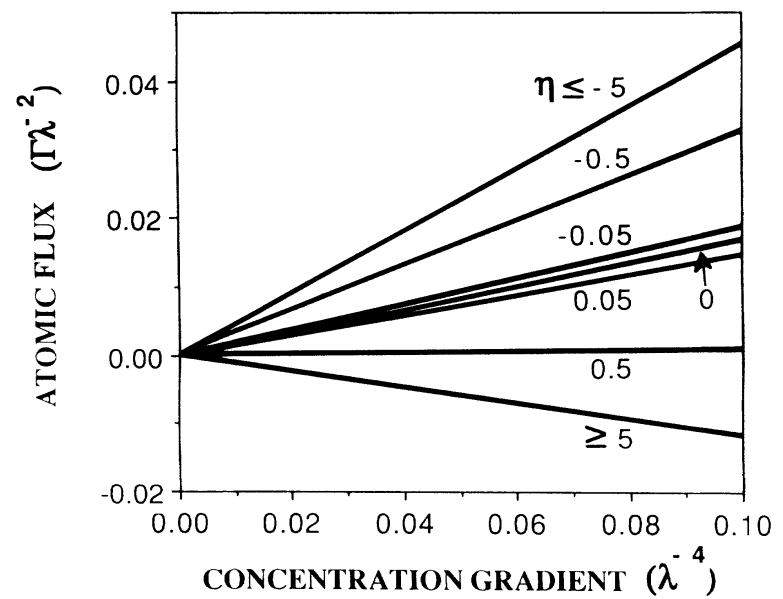

FIG. 1. The atomic flux as a function of the concentration gradient (in dimensionless units) calculated for different values of $\eta=\Delta H_{\mathrm{mix}} / k_{B} T$, as indicated. $\Gamma=6 v$ is the total atomic attempt frequency (in all directions). The lines for all $\eta \geq 5$ $(\leq-5)$ are nearly identical.

the present results.

The results illustrate the shortcoming of both the LR approximation and the MF theory. While the flux is nearly proportional to the concentration gradient for a given $\eta$, the LR assumption [Eq. (1)] would also require it to be linear in $\eta$ for a regular solution and a given concentration gradient. Considering the slopes of the lines in Fig. 1, this is clearly not the case. Figure 1 is in disagreement with the modified Tu expression [Eq. (6)], which predicts that the flux becomes independent of $\nabla C_{\alpha}$ for $\eta \rightarrow \infty$. Also, Eq. (6) predicts the validity of Darken's equation for sufficiently small composition gradients. The present results show, however, that the chemical enhancement saturates at equal values for arbitrarily small composition gradients. We conclude that the MF theory is not applicable in this limit. The physical reason for the saturation is the fact that for $|\eta| \gg 1$, all downhill jumps, and no uphill jumps, are successful. There is a negligible probability that a thermal fluctuation will cause an uphill jump or impede a downhill jump. This behavior is a result of the discrete spectrum of $\Delta \varepsilon_{i}$ 's and the fact that their occupations, but not the energy values, are a function of the composition gradient.

In Fig. 2, for sufficiently small values of $\eta$, the present results approach the linear behavior of Eq. (3). However, the slope of the calculated curve is approximately $16 \%$ greater than that of Eq. (3). This is a result of the fact that the regular-solution expression in Eq. (3) describes the evolution of an alloy in a subspace of parameter space in which the chemical short-range order (SRO) is zero. In the present model, however, although initially random, the evolution of the alloy is not constrained to a vanishing SRO and therefore occurs at a higher rate (each downhill jump increases the SRO). The effect of

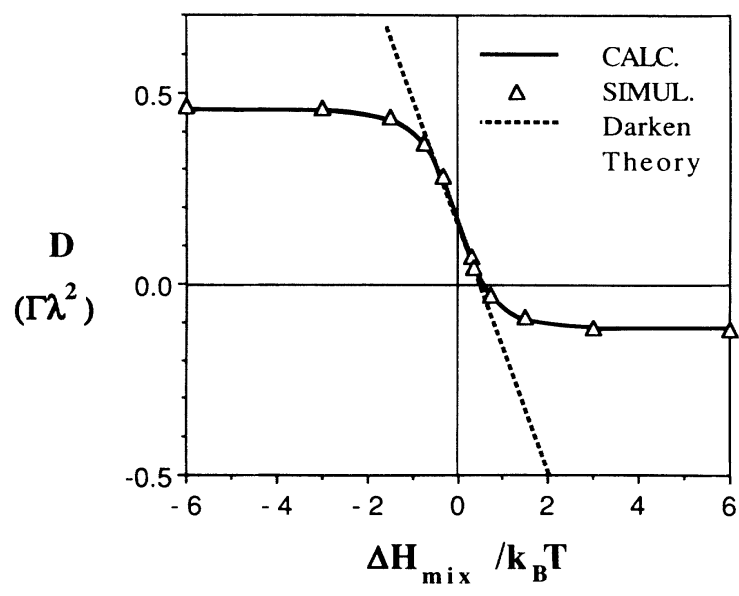

FIG. 2. The diffusion coefficient determined from the slopes in Fig. 1, as a function of $\eta=\Delta H_{\mathrm{mix}} / k_{B} T$. The simulation results and Darken's equation are plotted for comparison. The simulation error in $D$ is less than 0.01 .

SRO on $p_{i j}(\Delta \varepsilon)$ is outside the scope of this Letter and will be described elsewhere. ${ }^{19}$ Another aspect not included is the existence of correlation effects. ${ }^{9,20}$ Geometrical correlations exist for diffusion mechanisms such as vacancy or interstitialcy, but not for interstitial or exchange mechanisms. On the other hand, physical correlations between atomic jumps in alloys exist for all jump mechanisms. The author expects, however, the saturation of atomic-jump frequencies to be inherent to all mechanisms.

An experimental verification of the present theory is difficult because of the need to perform independent measurements of tracer- and chemical-diffusion coefficients. A measurement of the temperature dependence of the chemical-diffusion coefficient alone will not suffice because, given the large uncertainties in diffusion measurements, the exponential temperature dependence of $D_{a}^{*}$ will obscure the weaker temperature dependence of the thermodynamic factor. In addition, for large $\eta$ 's, chemical order or phase separation is likely to develop during interdiffusion. To the author's best knowledge, experimental verification of Darken's equation exists only for small values of $\eta .^{17,21}$ There are, however, diffusion geometries for which a MF theory makes the treatment one dimensional and therefore yields erroneous results. A discrete, analytical version ${ }^{22}$ of the theory of spinodal decomposition predicts a positive amplification factor for the evolution of a multilayered solid for $-\eta \gg 1$ and sufficiently small modulation wavelengths. In contrast, Monte Carlo simulations ${ }^{14}$ and experiments $^{23.24}$ show that for $\Delta H_{\text {mix }}<0$, if a modulation is inconsistent with long-range order, then the amplification factor is negative for all wavelengths. The present analytical treatment can be applied to this diffusion geometry and yield expressions for ordering kinetics. ${ }^{19}$ 
In summary, we have performed analytical and Monte Carlo calculations of the initial stages of chemical interdiffusion in a simple cubic, chemically random, binary alloy. For large enthalpies of mixing, the chemical enhancement of the diffusion coefficient is shown to saturate and be significantly lower than that previously predicted, even for infinitesimally small concentration gradients. It is concluded that neither the mean-field theory nor the linear-response approximation is applicable in this regime. Further work will include the effect of chemical order for alloys in equilibrium.

This work was supported by the National Science Foundation, Grant No. DMR-8820285. The author would like to thank Y.-T. Cheng and D. J. Srolovitz for reviewing the manuscript.

'M. Hillert, Acta Metall. 9, 525 (1961); J. W. Cahn, Acta Metall. 9, 795 (1961).

${ }^{2}$ L. S. Darken, Trans. AIME 175, 184 (1948).

${ }^{3}$ H. E. Stanley, Introduction to Phase Transitions and Critical Phenomena (Oxford Univ. Press, New York, 1971), p. 76.

${ }^{4} \mathrm{~S}$. R. de Groot and P. Mazur, Non-Equilibrium Thermodynamics (Dover, New York, 1984), Chap. 4.

${ }^{5}$ R. A. Swalin, Thermodynamics of Solids (Wiley, New York, 1972), pp. 131 and 145.

${ }^{6}$ Y.-T. Cheng, M. Van Rossum, M.-A. Nicolet, and W. L. Johnson, Appl. Phys. Lett. 45, 185 (1984).

${ }^{7}$ K. N. Tu, Annu. Rev. Mater. Sci. 15, 147 (1985).
${ }^{8}$ H. Bakker, in Diffusion in Crystalline Solids, edited by G. E. Murch and A. S. Nowick (Academic, Orlando, 1984), pp. 235-253.

${ }^{9}$ G. E. Murch, Philos. Mag. A 41, 157 (1980).

${ }^{10} \mathrm{~K}$. W. Kehr and K. Binder, in Applications of the Monte Carlo Method in Statistical Physics, edited by K. Binder, Topics in Current Physics Vol. 36 (Springer-Verlag, Berlin, 1984), p. 198.

${ }^{11} \mathrm{P}$. Shewmon, Diffusion in Solids (TMS, Warrendale, 1989), pp. 182-184.

${ }^{12}$ C. P. Flynn, Point Defects and Diffusion (Oxford Univ. Press, London, 1972), p. 393.

${ }^{13}$ E. O. Kirkendall, Trans. AIME 147, 104 (1942).

${ }^{14}$ M. Atzmon, J. Mater. Res. 5, 92 (1990).

${ }^{15}$ L. S. Darken and R. W. Gurry, Physical Chemistry of Metals (McGraw-Hill, New York, 1953), Chap. 19.

${ }^{16} \mathrm{~A}$ justification for the use of chemical rate equations was given in G. Martin, Phys. Rev. B 41, 2279 (1990).

${ }^{17} \mathrm{~J}$. C. Fisher, J. H. Hollomon, and D. Turnbull, Trans. AIME 175, 202 (1948).

${ }^{18}$ Flynn (Ref. 12), p. 20.

${ }^{19} \mathrm{M}$. Atzmon (to be published).

${ }^{20}$ Shewmon (Ref. 11), p. 107.

${ }^{21}$ C. E. Birchenall and R. F. Mehl, Trans. AIME 171, 143 (1947).

${ }^{22}$ H. E. Cook, D. de Fontaine, and J. E. Hilliard, Acta Metall. 17, 765 (1969).

${ }^{23}$ W. M. Paulson and J. E. Hilliard, J. Appl. Phys. 48, 2117 (1977)

${ }^{24} \mathrm{M}$. Atzmon and F. Spaepen (unpublished results on interdiffusion in amorphous $\mathrm{Ni}_{55} \mathrm{Zr}_{45}$ ). 